\title{
Modeling Correlated and Uncorrelated Distortion in Communication Systems
}

\author{
Frank P. Hart*, Nuno B. Carvalho ${ }^{\dagger}$, Kevin G. Gard*, and Michael B. Steer* \\ *Department of Electrical and Computer Engineering \\ North Carolina State University, Raleigh, North Carolina 27695-7914 \\ Email: fphart@eos.ncsu.edu,kggard@ncsu.edu,mbs@ncsu.edu \\ $\dagger$ Instituto de Telecommunicações, Universidad de Aveiro, 3810-193 Aveiro, Portugal \\ E-mail: nbcarvalho@ua.pt
}

\begin{abstract}
Simulation technology suitable for extracting correlated and uncorrelated distortion in communication systems is presented. This is achieved through a new frequencyindexing scheme with the Arithmetic Operator Method that separately tracks correlated and uncorrelated intermodulation products when multi-tone signals with uncorrelated phase are amplified through a nonlinear system. The indexing scheme is illustrated with a three-tone example and results from a fifth-order nonlinear amplifier excited by fifteen independent tones are considered. In the example it is shown that undesirable uncorrelated in-band distortion is lower than the innocuous correlated in-band distortion.
\end{abstract}

Index Terms-Multi-tone signals, uncorrelated phase, nonlinear amplifiers, computer-aided analysis, co-channel distortion, in-band distortion

\section{INTRODUCTION}

In recent years it has become apparent that uncorrelated intermodulation (IM) distortion components appearing in the same frequency band as the amplified output are the major contributors to performance degradation of microwave power amplifiers. Correlated IM is a minute inphase degradation of the linearly amplified output signal and has no impact on the performance of a system. Correlated IM can be viewed as the multi-tone equivalent of gain compression or enhancement rather than as signal impairment. Uncorrelated IM, however, is viewed as undesirable and is included as distortion in the signal-tointerference, noise, and distortion (SINAD) ratio. Unfortunately, commercial simulation environments, by the inherent nature of their time-domain nonlinear solvers, enforce correlation of frequency domain artifacts that appear at the same numerical frequency, thus making it impossible to separate correlated IM from uncorrelated IM. Furthermore, recently-published results [1] have shown that correlated IM occurs at higher power levels than uncorrelated IM. It is easy to speculate that commercial product designs have been completed with SINAD ratios that included the correlated IM in the ratio's denominator, thus exceeding the necessary SINAD ratio specification at the expense of other design parameters or resulting in components that were more costly than necessary. The shortcomings of enforced correlation in commercial environments has spurred researchers to develop analytical and simulation methods to distinguish correlated and uncorrelated IM along with laboratory measurement schemes for separating these two forms of distortion.

On the analytical front, researchers at the Universidad de Aveiro have developed methods for predicting power levels of correlated and uncorrelated IM products in the cochannel band [1]. The methodology combines the use of 3rd order Volterra transfer function models including memory and cross-correlation post-processing of the results of time-domain or Harmonic Balance simulations. The work is the culmination to date of analytical work that began on uncorrelated multi-tones several years ago [2]. At that time, the analytical results were validated through a modification of a previously-disclosed simulation environment [3], [4] that enforced use of equally-spaced frequencies, thus eliminating the possibility of storing output spectral content of differing input frequency mixtures that mapped to the same output frequency. However, the response to uncorrelated multi-tones was computed at each frequency during the mixing computations, thus giving the correct output spectra without attempting to separate correlated and uncorrelated IM. In [5], [6] Gharaibeh, Gard, and Steer developed and disclosed a methodology for analyzing and measuring the correlated and uncorrelated co-channel band distortion produced by a multisine representation of a CDMA signal. Boulejfen et al [7] developed memoryless 5th order Volterra transfer functions (and methods for analyzing them) and implemented them in commercial simulators with good results, but no attempt was made to distinguish correlated and uncorrelated IM output power in the co-channel band.

Here, for the first time, a frequency-domain simulation environment (called the AOM Toolbox) is used to predict in-band correlated and uncorrelated IM power levels. The separation of correlated and uncorrelated power data is facilitated by simple inspection of the Basic Intermodulation Product Description (BIPD) table, a table of vectors that forms an underlying vector decomposition of the numerical frequency domain such that statistically orthogonal output frequency mixtures occurring at the same numerical fre- 
quency are assigned to unique phasors in the vectors used to compute the output spectra. The predicted correlated and uncorrelated power is computed from stored data that was previously used to demonstrate that the AOM Toolbox accurately predicts the response of a 5 th order nonlinearity to a 15-tone uncorrelated phase source [8].

\section{Construction of a Three-Tone BIPD Table}

Consider three unit-amplitude independent tones, $f_{1}, f_{2}$, and $f_{3}$ located at 449,450 , and $451 \mathrm{MHz}$, respectively, and let them have identically distributed random phases $\phi_{1}, \phi_{2}$, and $\phi_{3}$, respectively. Expressed in a compact form,

$$
x(t)=\frac{1}{2} \sum_{q=1}^{3}\left[e^{\jmath \phi} e^{\jmath 2 \pi f_{q} t}+e^{-\jmath \phi} e^{-\jmath 2 \pi f_{q} t}\right],
$$

where $\jmath \equiv \sqrt{-1}$ and $x(t)$ has the Fourier Transform

$$
X(f)=\frac{1}{2} \sum_{q=1}^{3}\left[e^{\jmath \phi} \delta\left(f-f_{q}\right)+e^{-\jmath \phi} \delta\left(f+f_{q}\right)\right] .
$$

Now suppose $x(t)$ is passed through a memoryless nonlinear transfer function limited to the 3rd order, so,

$$
y(t)=\sum_{n=1}^{3}\left[a_{n}[x(t)]^{n}\right]
$$

and $y(t)$ has the Fourier Transform

$$
\begin{aligned}
Y(f)= & a_{1} X(f)+a_{2} X(f) * X(f) \\
& +a_{3} X(f) * X(f) * X(f),
\end{aligned}
$$

where $*$ denotes convolution. Since $X(f)$ is comprised of Dirac delta functions, the form of the output signal will be comprised of Dirac delta functions at frequency mixtures of two tones at the second order and three tones at the third order. If the input frequencies are assigned to a column vector $\mathbf{f}_{i n}=\left[\begin{array}{lll}f_{1} & f_{2} & f_{3}\end{array}\right]^{\mathrm{T}}$ and the weightings of the input frequencies appearing at the output are expressed as a row vector $\boldsymbol{\eta}_{k}^{\mathrm{T}}=\left[\begin{array}{lll}\eta_{k, 1} & \eta_{k, 2} & \eta_{k, 3}\end{array}\right]$, then a compact expression for the numerical frequency locations of the output spectra is given by the dot product of $\boldsymbol{\eta}_{k}^{\mathrm{T}} \cdot \mathbf{f}_{i n}$, where the permissible frequency weightings in $\boldsymbol{\eta}_{k}^{\mathrm{T}}$ are determined by the discrete frequency convolution in (4). Tabulating these frequency weightings is a combinatorial problem at each order of nonlinearity, but construction of the table is relatively straightforward, and for real signals of the sort used here, the conjugate symmetry of the spectral content permits a simplification of the table to DC and positive frequencies, i.e. a one-sided spectrum.

The construction process begins with the linear response, which is given simply by entering the rows of a 3 by 3 identity matrix as the first entries of the table. Next, the second order response is determined by adding and subtracting the span of the 3 by 3 space of upper circulant shift matrices to the linear response. Thus the matrices added and subtracted to the linear response are:

$$
\begin{aligned}
& {\left[\begin{array}{lll}
0 & 1 & 0 \\
0 & 0 & 1 \\
1 & 0 & 0
\end{array}\right] \quad\left[\begin{array}{lll}
0 & 0 & 1 \\
1 & 0 & 0 \\
0 & 1 & 0
\end{array}\right] \quad\left[\begin{array}{lll}
1 & 0 & 0 \\
0 & 1 & 0 \\
0 & 0 & 1
\end{array}\right]} \\
& \text { Unit shift Two shifts Three shifts }
\end{aligned}
$$

The Unit shift matrix is the basic upper circulant matrix; when multiplied by itself, it gives the Two shifts matrix, and a further multiplication by the Unit shift matrix yields the Three shifts or identity matrix. Adding and subtracting the Unit shift and Two shifts matrices to the identity matrix for the linear response creates candidate matrices of BIPD table entries that correspond to IM products, while adding the Three shifts matrix yields harmonic products. Row vectors from these candidate matrices are added to the BIPD table if:

- The 1-norm of the row vector is equal to the current nonlinear order under construction

- The numerical frequency of the row vector is nonnegative

- The row is not a duplicate of an existing table entry The result of the construction process for the second order response is the addition of the rows of the Second Order matrix below to the BIPD Table.

$$
\left[\begin{array}{ccc}
-1 & 0 & 1 \\
-1 & 1 & 0 \\
0 & -1 & 1 \\
2 & 0 & 0 \\
0 & 2 & 0 \\
0 & 0 & 2 \\
1 & 1 & 0 \\
0 & 1 & 1 \\
1 & 0 & 1
\end{array}\right]
$$

Second Order

$$
\left[\begin{array}{ccc}
-1 & 2 & 0 \\
0 & -1 & 2 \\
-1 & 1 & 1 \\
1 & -1 & 1 \\
-1 & 0 & 2 \\
3 & 0 & 0 \\
0 & 3 & 0 \\
0 & 0 & 3 \\
2 & -1 & 0
\end{array}\right]
$$

Third Order

$$
\left[\begin{array}{ccc}
0 & 2 & -1 \\
2 & 1 & 0 \\
0 & 2 & 1 \\
1 & 0 & 2 \\
2 & 0 & -1 \\
2 & 0 & 1 \\
1 & 2 & 0 \\
0 & 1 & 2 \\
1 & 1 & -1 \\
1 & 1 & 1
\end{array}\right]
$$

Third Order
The third order response portion of the BIPD table, shown above and to the right (as two matrices), is constructed from the second order portion through application of the same process used to construct the second order response from the linear response. The all-zero DC row vector is prepended to complete construction of the BIPD table, and the resulting table contains 32 rows of vectors, each containing 3 elements and representing a unique possible mixing output. The fully constructed the BIPD table is sorted by increasing 1-norm, not by frequency. Before its further use in the AOM Toolbox, the BIPD table is sorted by numerical frequency, with the result in Table I.

Note that the AOM Toolbox algorithm that constructs the BIPD table is not limited to three tones and a 3rd order 
nonlinearity. Extension to greater numbers of arbitrarilyspaced input tones and higher orders of nonlinearity is straightforward and has been implemented in Matlab $^{\circledR}$. While the algorithm is practically bound by combinatorial complexity as described in [8], it has computed the BIPD table for a 220 tone input through a 3rd order nonlinearity in about two hours while 100 tones through a 3rd order nonlinearity takes about 4 minutes.

\section{DATA Mining the BIPD TABle Illustrated}

Notice that each row of Table I contains a unique vector of frequency weightings, but numerical frequencies are not unique. This poses no problem to the AOM Toolbox simulation environment, which maintains a 1-to-1 relationship between the number of rows in the BIPD table and the number of phasors in vectors used to compute output spectra. The convolutions in (4) are performed via matrixvector multiplications where the row and column spaces of the matrices also maintain a 1-to-1 phasor relationship with the number of rows in the BIPD table.

Focusing on the co-channel band frequencies, i.e. the rows at Frequency Indices 7 through 12 in Table I, note that the unit vector entries at indices 7, 9, and 11 correspond to the input (and linear output) frequencies while the entries at indices 8, 10, and 12 correspond to in-band 3rd order IM products at the same respective frequencies. Consider Frequency Indices 7 and 8 first. Here, the IM term at index 8 is composed of a mixture of twice $f_{2}$ minus $f_{3}$. Since $f_{1}$ does not appear $\left(\eta_{8,1}=0\right)$, the IM term at index 8 is statistically independent and therefore uncorrelated [9] from the input at index 7. A similar observation can be made about the IM term at index 12 relative to the input at index 11 . Turning to Frequency Indices 9 and 10, notice that the IM term at Index 10 is a mixture of all three input frequencies and specifically $\eta_{10,2}=-1$, so that this IM term statistically depends upon $f_{2}$. However, a correlation analysis, assuming zeromean uniformly distributed random variables for the input phases, yields a result of 0 for the cubic function of the complex exponentials producing the IM term at frequency index 10 with the linear term at frequency index 9. Thus, although the random phase variables are statistically dependent, the spectral content created is orthogonal and uncorrelated. In general, the spectral content at each BIPD is orthogonal and uncorrelated from the other BIPDs.

Since the BIPD table shows all possible output frequency combinations, it follows (and can be shown by correlation analysis) that correlated components occur only when a second-order IM term in the BIPD Table $\left(\left\|\boldsymbol{\eta}_{k}\right\|_{1}=\right.$ 2 ) is mixed (BIPDs are added or subtracted) with one of the inputs $\left(\left\|\boldsymbol{\eta}_{k}\right\|_{1}=1\right)$ such that the resulting BIPD corresponds to an input. Such mixtures necessarily involve phase cancellation. For example, adding the BIPDs at frequency indices 7 and 1 yields the BIPD at frequency
TABLE I

ILLUSTRATIVE FREQUENCY-SORTED BIPD TABLE

\begin{tabular}{|c|c|c|c|c|c|}
\hline $\begin{array}{c}\text { Freq. } \\
\text { Index } \\
k\end{array}$ & $\begin{array}{c}1- \\
\text { Norm } \\
\left\|\boldsymbol{\eta}_{k}\right\|_{1}\end{array}$ & $\begin{array}{c}f_{1} \\
\text { Weight } \\
\eta_{k, 1}\end{array}$ & $\begin{array}{c}f_{2} \\
\text { Weight } \\
\eta_{k, 2}\end{array}$ & $\begin{array}{c}f_{3} \\
\text { Weight } \\
\eta_{k, 3}\end{array}$ & $\begin{array}{l}\text { Num. } \\
\text { Freq. } \\
\text { (MHz) }\end{array}$ \\
\hline 0 & 0 & 0 & 0 & 0 & 0 \\
\hline 1 & 2 & -1 & 1 & 0 & 1 \\
\hline 2 & 2 & 0 & -1 & 1 & 1 \\
\hline 3 & 2 & -1 & 0 & 1 & 2 \\
\hline 4 & 3 & 2 & 0 & -1 & 447 \\
\hline 5 & 3 & 2 & -1 & 0 & 448 \\
\hline 6 & 3 & 1 & 1 & -1 & 448 \\
\hline 7 & 1 & 1 & 0 & 0 & 449 \\
\hline 8 & 3 & 0 & 2 & -1 & 449 \\
\hline 9 & 1 & 0 & 1 & 0 & 450 \\
\hline 10 & 3 & 1 & -1 & 1 & 450 \\
\hline 11 & 1 & 0 & 0 & 1 & 451 \\
\hline 12 & 3 & -1 & 2 & 0 & 451 \\
\hline 13 & 3 & 0 & -1 & 2 & 452 \\
\hline 14 & 3 & -1 & 1 & 1 & 452 \\
\hline 15 & 3 & -1 & 0 & 2 & 453 \\
\hline 16 & 2 & 2 & 0 & 0 & 898 \\
\hline 17 & 2 & 1 & 1 & 0 & 899 \\
\hline 18 & 2 & 0 & 2 & 0 & 900 \\
\hline 19 & 2 & 1 & 0 & 1 & 900 \\
\hline 20 & 2 & 0 & 1 & 1 & 901 \\
\hline 21 & 2 & 0 & 0 & 2 & 902 \\
\hline 22 & 3 & 3 & 0 & 0 & 1347 \\
\hline 23 & 3 & 2 & 1 & 0 & 1348 \\
\hline 24 & 3 & 2 & 0 & 1 & 1349 \\
\hline 25 & 3 & 1 & 2 & 0 & 1349 \\
\hline 26 & 3 & 0 & 3 & 0 & 1350 \\
\hline 27 & 3 & 1 & 1 & 1 & 1350 \\
\hline 28 & 3 & 0 & 2 & 1 & 1351 \\
\hline 29 & 3 & 1 & 0 & 2 & 1351 \\
\hline 30 & 3 & 0 & 1 & 2 & 1352 \\
\hline 31 & 3 & 0 & 0 & 3 & 1353 \\
\hline
\end{tabular}

index $9\left(\left[\begin{array}{lll}1 & 0 & 0\end{array}\right]+\left[\begin{array}{lll}-1 & 1 & 0\end{array}\right]=\left[\begin{array}{lll}0 & 1 & 0\end{array}\right]\right)$, which is an input frequency, while subtracting the BIPD at index 7 from the BIPD at index 17 also yields the input frequency at index $9\left(\left[\begin{array}{lll}1 & 1 & 0\end{array}\right]-\left[\begin{array}{lll}1 & 0 & 0\end{array}\right]=\left[\begin{array}{lll}0 & 1 & 0\end{array}\right]\right.$, similarly when index 2 is subtracted from index 11, when index 9 is subtracted from index 18, and when index 11 is subtracted from index 20 , respectively). The convolution operations produce this correlated (vectorially-added) result in the spectral vector for the 3rd order output phasor (thus keeping it separate from the linear output) corresponding to frequency index 9.

\section{RESUlts AND Discussion}

The method for distinguishing correlated and uncorrelated in-band IM distortion described in Sec. III was applied to the previous results of simulations that were in good agreement with measurements of a microwave amplifier response to 15 independently-generated tones [8]. The power of the uncorrelated IM terms occurring in the co- 


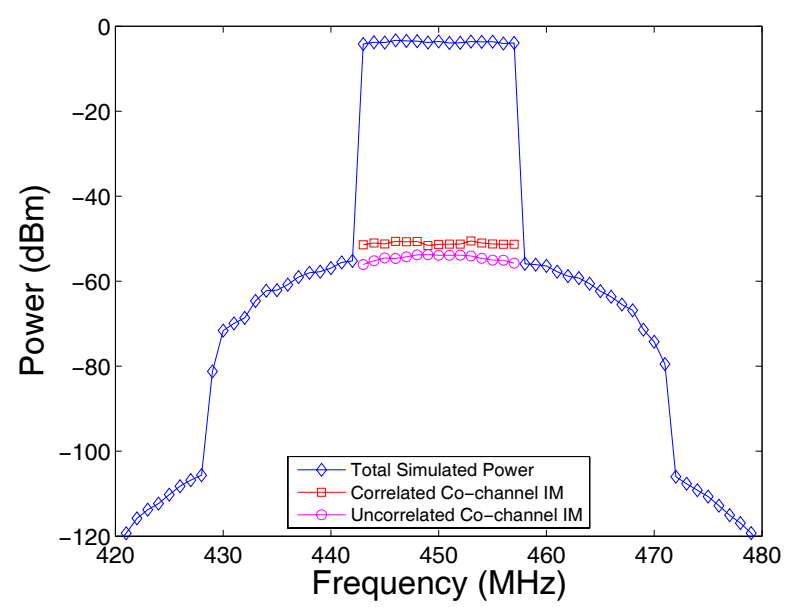

(a) Adjacent and Co-channel band power.

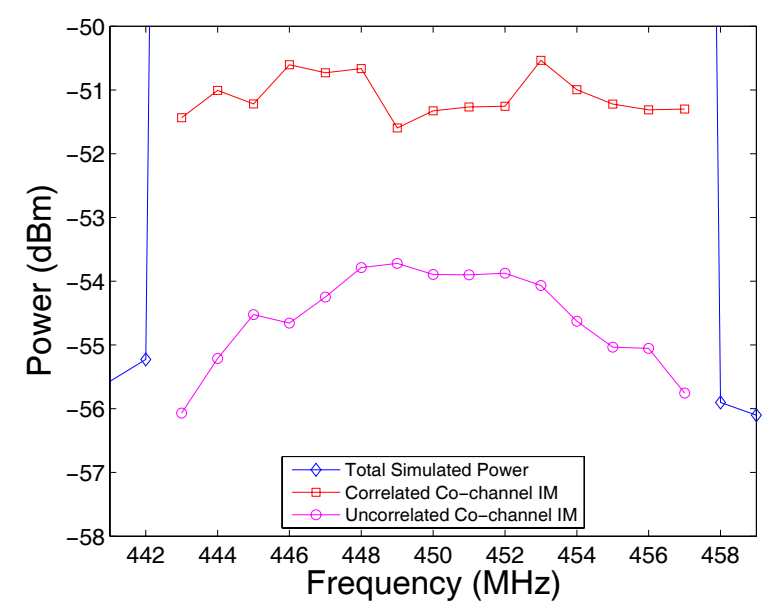

(b) Magnification of the Co-channel band.

Fig. 1. Results of mining the BIPD table to separate correlated and uncorrelated co-channel power.

channel band was computed by summing the squared magnitudes of the orthogonal phasors from a single simulation, and the power of the correlated IM terms was computed by performing an averaging of the squared magnitudes of the results of 30 simulations, with each simulation having a different set of random input phases. Figure 1(a) shows the total output power along with the correlated and uncorrelated co-channel IM power while Fig. 1(b) provides a magnified view of the co-channel power band. Good agreement can be seen between the results predicted here and those obtained by applying analytical crosscorrelation methods to a memoryless nonlinearity in [1]. Specifically, the correlated power is several $\mathrm{dB}$ higher than the uncorrelated power at each frequency in the co-channel band. In [1], the correlated power is flat across the band, while here there is a $1.5 \mathrm{~dB}$ variation. This is due to the fact that there is a $0.6 \mathrm{~dB}$ variation in the amplitudes of the 15-tone physical source used and an approximate 0.2 $\mathrm{dB}$ in-band loss variation in the cable and combiner chain (and reflected in the simulation environment here) from the sources to the amplifier input, while in [1] the sources were of equal amplitude.

\section{CONCLUSiON}

Simulation data which were previously used to validate the AOM Toolbox against measurements have been easily mined to predict the in-band correlated and uncorrelated IM power, and agreement was seen between these predictions and those predicted for memoryless nonlinearities using analytical methods. The key to facilitating the data mining is the BIPD table, which orthogonally decomposes the numerical frequency domain such that the spectra for correlated and uncorrelated outputs are stored at different locations.

\section{ACKNOWLEDGMENT}

This material is based upon work supported by the U.S. Army Research Laboratory and the U.S. Army Research Office as a Multidisciplinary University Research Initiative on Standoff Inverse Analysis and Manipulation of Electronic Systems under grant number W911NF-05-1-0337.

\section{REFERENCES}

[1] J. Martins, N. B. Carvalho, and J. C. Pedro, "Intermodulation distortion of third-order nonlinear systems with memory under multisine excitations," IEEE Transactions on Microwave Theory and Techniques, vol. 55, no. 6, pp. 1264-1271, June 2007.

[2] J. C. Pedro and N. B. de Carvalho, "On the use of multitone techniques for assessing rf components' intermodulation distortion," IEEE Transactions on Microwave Theory and Techniques, vol. 47, no. 12, pp. 2393-2402, December 1999.

[3] N. B. de Carvalho and J. C. Pedro, "Simulating strong non-linear microwave circuits driven by a large number of input tones," in 27 th European Microwave Conference and Exhibition, vol. 2, 1997, pp. $820-825$.

[4] _ _ "Multitone frequency-domain simulation of nonlinear circuits in large- and small-signal regimes," IEEE Transactions on Microwave Theory and Techniques, vol. 46, no. 12, pp. 2016-2024, December 1998.

[5] K. M. Gharaibeh, K. G. Gard, and M. B. Steer, "Estimation of inband distortion in digital communication system," in 2005 IEEE MTT-S International Microwave Symposium Digest, Long Beach, CA, June 12-17, 2005.

[6] K. M. Gharaibeh, K. Gard, and M. B. Steer, "In-band distortion of multisines," IEEE Transactions on Microwave Theory and Techniques, vol. 54, no. 8, pp. 3227-3236, August 2006.

[7] N. Boulejfen, A. Harguem, and F. H. Ghannouchi, "New closed-form expressions for the prediction of multitone intermodulation distortion in fifth-order nonlinear rf circuits/systems," IEEE Transactions on Microwave Theory and Techniques, vol. 52, no. 1, pp. 121-132, January 2004.

[8] F. P. Hart and M. B. Steer, "Modeling the nonlinear response of multitones with uncorrelated phase," IEEE Transactions on Microwave Theory and Techniques, vol. 55, no. 10, pp. 2147-2156, October 2007.

[9] P. Z. Peebles, Jr., Probability, Random Variables, and Random Signal Principles, 4th ed. McGraw-Hill, 2001. 